International Journal of Engineering \& Technology, $7(4.36)(2018)$ 1079-1082
International Journal of Engineering \& Technology
SPC
Website: www.sciencepubco.com/index.php/IJET
Research paper

\title{
Main Approaches and Stages of Prototyption: the Example of Designing and Creating Automatic System for Fabric Transfer Patterns
}

\author{
V.V. Moroz ${ }^{1}$, L.V. Baulina ${ }^{2}$ \\ ${ }^{1}$ Prototyping center of Dubna State University, Russia, Dubna \\ ${ }^{2}$ Prototyping center of Dubna State University, Russia, Dubna \\ *Corresponding authorE-mail: moroz@uni-dubna.ru
}

\begin{abstract}
This article gives a detailed description of the main approaches and the sequence of manufacturing prototypes of new devices by the example of creating a new device - an automatic patterning system for fabric. Both amateur and industrial ways of solving this problem are described. The basic steps of development of the device from the description of idea, definition of purposes and tasks of development, before creation of the prototype are resulted. Description of the stages of virtual modeling and visualization, the main methods and principles of prototype creation are also presented.
\end{abstract}

Keywords: synthesis of technical systems, morphological methods, method Saati, analysis of requirements for the device.

\section{Introduction}

Nowadays, household automation is a a means of solving problems as a partner of a person under the motto "Technique with a man for a man" [1] and also contributes to the improvement of life quality - it performs the corresponding man-machine functions with the help of human-integrated technical systems. Consider task of automating the process of both mass and individual tailoring.

The main problem is that although process of creating patterns (with the help of sewing CAD systems [2-5]) and sewing (with the help of sewing machines) are automated, the process of marking fabric in living conditions is performed, in most cases, manualy and inefficiently.

\section{Industrial technologies}

To perform the cutting-preparatory work at the sewing enterprises, a number of equipment is used as following, which allows to simplify and speed up the process [6-8]:

The computer-aided design system (CAD) solves the tasks of automating work at the stages of designing and preparing patterns - automating the design and technological preparation of clothing production.

Plotter for the preparation of patterns - draws a pattern on paper and cuts it.

Device for flooring, laying fabrics for the formation of several layers.

The cutting machine makes cutting either automatically or automatically using disk / ribbon knives.
Marking parts cut. It is carried out with the help of chalk, laying on the details of cutting patterns, as well as using a long needle for puncturing the depth of the flooring.

Industrial equipment is not suitable for home use for a number of reasons: dimensions, high cost, narrow specialization of equipment.

Technologies used in everyday life

n everyday life, sewing enthusiasts often use CAD systems (for example, AutoCAD) to create patterns - the only automated step. Further, to obtain patterns, the following operations are performed: the patterns are divided into several A4 sheets, marked sheets, printed, glued all the sheets in the desired sequence to obtain a full-sized pattern. After that, the pattern is cut out, put on the prepared fabric and outlines the contours. Now we can say that the pattern is applied to the fabric. Cut out the fabric from the fabric and thus get the details of the cut [9].

\section{Formulation of the problem}

Consider the task of automating listed manual, routine operations to obtain cut parts for convenient use at home.

First of all, it is necessary to determine the requirements for the system, determine the source data, the results of the work. The easiest way to do this is in the form of diagrams describing the system. The initial description of the system using the graphical notation of functional modeling - IDEF0 $[10,11]$ is best suited for such a representation of the system, identifying requirements and constraints.

The description looks like a "black box" with inputs, outputs, control actions, a mechanism that is gradually detailed to the required level. Those. notation prescribes the construction of a hierarchical system of diagrams. In order to be properly understood, there are dictionaries describing activities and arrows. 
In these dictionaries you can give descriptions of what meaning is embedded in each activity or arrow.
Figure 1 shows the IDEF0 diagram, which demonstrates the basic requirements for system inputs and outputs and general system requirements.

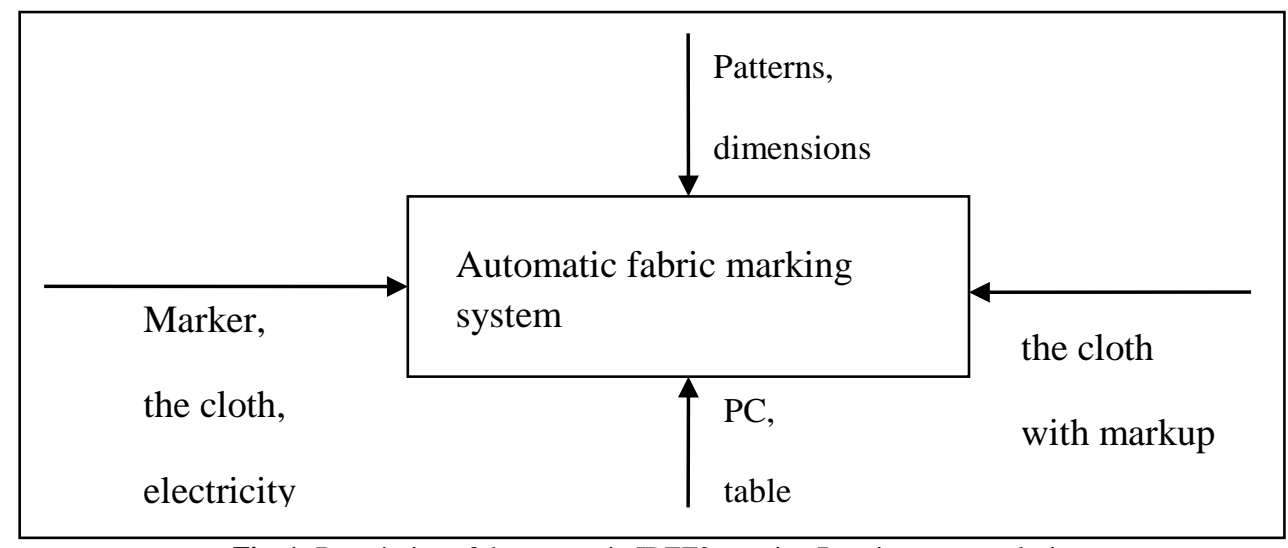

Fig. 1: Description of the system in IDEF0 notation Requirements analysis

To analyze the system requirements, we use the classification of requirements for the FURPS + systems [12]. Acronym refers to the following categories of requirements:

Functionality - functional. The system should allow you to download files with patterns, provide an opportunity to observe the marking process, stop the marking process, resume from the moment it was stopped, or from the last drawn element, start the marking.

Usability - usability. The system should be easy to operate. Must be ergonomic - should not require long training. A working system should not create strong noises. The device should be compact, not take up much space, both during storage and during operation.

Reliability requirements.

Performance - performance requirements. The execution of markup in a time less than or equal to the time that a person spends when translating the pattern on the fabric in the domestic environment. Compliance with accuracy: $\pm 5 \mathrm{~mm}$.

Supportability - support requirements.

And:

- Project restrictions. The limitation on the cost of the element base is the use of the minimum budget, or the use of existing components.

- Performance requirements. The system should be as simple as possible with simple support and as simple as possible software.

- Interface requirements. Simple, intuitive and user-friendly interface.

Additionally, the requirements of universal design [13] were considered: equality in use, easily perceived information, error admissibility.

\section{Searching of decisions}

As a rule, in engineering practice, there are no methods that allow to design the optimal structure of the device according to the terms of a technical task.

When describing system requirements, ideas appear that require fixation and initial performance testing. This approach is called the brainstorming method and is used in many areas of activity. Any of the proposed ideas may be the beginning of the development of a unique device. To broaden the search for a solution field, a search was also made for possible existing solutions to such problems - existing robots were considered that allow building drawings on various surfaces [14].

As a result of defining the requirements for the system, the initial representations of the appearance, the necessary elemental base required, the initial sketches and models are formed. In our case, we have prepared and considered several options for implementing the system.

To choose from several alternative solutions described, it is convenient to apply the hierarchy analysis method - the Saaty method. The essence of the method consists in choosing one of the alternatives by means of a weighted evaluation of the criteria [15]. Scales are scales of relationships. If according to any of the criteria there is an objective assessment, then they are normalized so that their sum is equal to one. If there is no such estimate, the method recommends using pair-wise comparisons. For this, the scale of pairwise comparisons is used: 1 - equivalence, 3 moderate superiority, 5 - strong superiority, 7 - very strong superiority, 9 - higher superiority. It is necessary to compare the solutions in pairs and set the ratio in the form of a fraction. Simple fractions are interpreted as: if, for example, at the intersection of the first row and second column there is a simple 4/1 fraction, this means that the first object is 4 times larger than the second object by the current criterion.

It is necessary to compare all objects by all criteria. Thus, we obtain a table of results of pairwise comparisons. Fractions are converted to decimal and the amounts are calculated line by line for each object, normalized to their sum was equal to one. Values are estimates for each criterion. Table 1 demonstrates the comparison by the criterion "compactness" - the receipt of the evaluation of alternatives is described.

Table 1: Results of the comparison according to the criterion "Compactness"

\begin{tabular}{|c|c|c|c|c|c|c|c|}
\hline & $\mathrm{I}$ & II & III & IV & $\mathrm{V}$ & $\sum$ & Total \\
\hline $\mathrm{I}$ & $1 / 1$ & $1 / 6$ & $1 / 3$ & $1 / 4$ & $1 / 7$ & 1,89 & 0,04 \\
\hline II & $6 / 1$ & $1 / 1$ & $1 / 3$ & $1 / 5$ & $1 / 1$ & 8,53 & 0,18 \\
\hline III & $3 / 1$ & $3 / 1$ & $1 / 1$ & $1 / 2$ & $1 / 4$ & 7,75 & 0,16 \\
\hline IV & $4 / 1$ & $5 / 1$ & $2 / 1$ & $1 / 1$ & $1 / 5$ & 12,2 & 0,25 \\
\hline $\mathrm{V}$ & $7 / 1$ & $1 / 1$ & $4 / 1$ & $5 / 1$ & $1 / 1$ & 18 & 0,37 \\
\hline & & & \multicolumn{3}{|c|}{ Amount: } & 48,37 & 1 \\
\hline
\end{tabular}

The criteria are compared in the same way: ease of implementation, implementation time, simplicity of software, cost of components, etc. Criteria are compared with each other in terms of their relative importance. Similarly, the resulting weights for each criterion are obtained.

The choice is made on the basis of the final estimates of alternatives - the utility function. To calculate it, a weighted sum of criteria is used, which is the sum of the products for each criterion - the weight of the criterion and the values of the criterion. For each of the options we get the value of the utility function. According to the Saaty method, the variant with the maximum value of the utility function is taken as the optimal variant. As a result of the choice of the best from the point of view 
of the listed requirements and characteristics, a device called "autoseater" was chosen.

Below is a description of the expected process of operation of the device.

The device moves in a well-fixed fabric and translates the drawings. Two main wheels and an extra ball wheel required for stability should be used for movement. The device applies markings with a marker on the fabric. The device is placed on a flat surface on the fabric, after which it begins to move along the fabric. To build a line, the device drives up to its beginning, lowers the marker, travels a certain distance with the marker lowered, and raises the marker. Next, the device approaches the beginning of the next line and the process repeats.

The implementation of the described solution must begin with the design. The first design stage is the creation of an architecture that satisfies the system requirements and solves the main task.

\section{Synthesis task}

To implement the described system, it is necessary: determine the basis of the electronic components used, solve the problem of their layout and geometric design, start implementing the software, make a prototype system. Proceeding from the description of the device, its appearance was given, shown in fig. 2

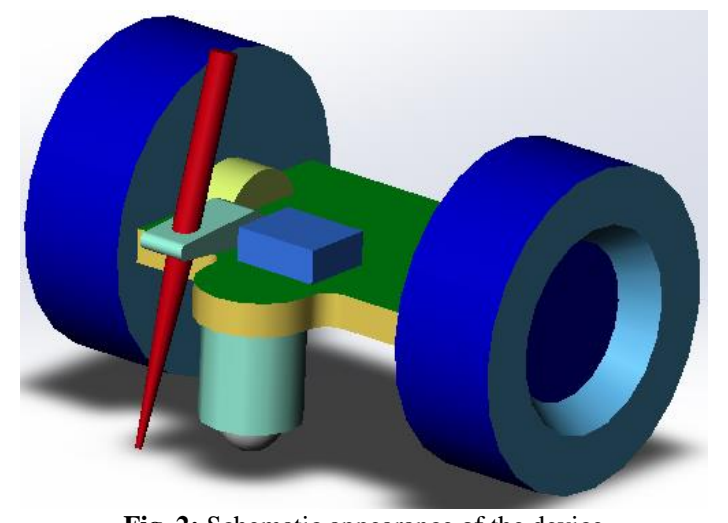

Fig. 2: Schematic appearance of the device

The model shown is not final and may be revised and amended. In the future should be added to the body and other elements.

To synthesize a specific instrument, it is necessary to select electronic, mechanical components from a huge variety of existing (or possible for manufacturing).

For the synthesis of a specific instrument, we use the morphological method [16]. In essence, it is necessary to determine the space for the search, including the desired solution, and then narrow this space by performing a search. In this case, the search space is called a morphological set, the process of determining the space is morphological analysis, and the search for a solution is called a morphological synthesis. According to the results of morphological analysis, a morphological set (set of alternatives) is determined. Synthesis is the search for the desired device on the morphological set.

The first stage of morphological analysis is the definition of classes of objects: microcontrollers, motors, wheels, markers, as well as the allocation of classification features for each class. For example, for motors it is voltage, current, spindle diameter, etc. A morphological table is compiled, each containing combinations of all elements according to appropriate classification features.

The results of the morphological analysis can be arranged in the form of a morphological tree or graph, as a rule, with many alternative solutions [17]. Here, the choice of a solution is affected by the availability of items, cost, and other parameters. Next, along the chosen chain, the layout design and prototype manufacturing is performed.
It is also necessary to solve the following tasks: to create a specific printed circuit board with suitable wiring [18,19], since such a board is device-specific, to create a housing and marker fixing elements [20].

\section{Prototype}

The process of creating the first functioning prototype is quite complicated.

All the steps described in the article (even the choice of implementation options) starting from the initial sketch of the expected device up to the manufacture of all elements and software are performed cyclically. You may need to change any elements in the model, in the printed circuit board, which often leads to a change in all other components. As a result of a certain number of iterations, a functioning prototype of the device is obtained, shown in Figure 3.

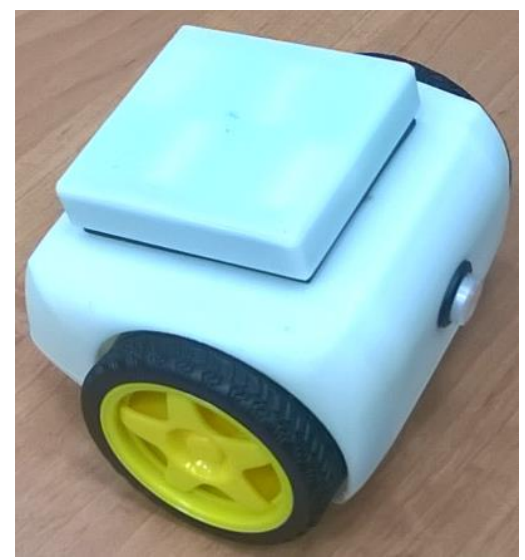

Fig. 3: Prototype device

In order to understand whether the required device has been received, it is necessary to compare it with the initial requirements for the device at the time of design. To do this, a procedure is tested.

As a rule, the device meets the requirements, since it was originally designed to meet them. However, most often there are new requirements and suggestions for refinement. The prototype of the implemented device has been tested.

\section{References}

[1] Breido I.V. History, state and prospects of development of automation. Karaganda State Technical University. - Karaganda, 2016.

[2] Truevtseva M.A., Evgenyeva A.M., Filina I.N. Development of information support of computer-aided design systems for technological processes of sewing shops of service enterprises. Clothing industry, 2014. - № 2. S. 28-31.

[3] Surikov G.I., Surikov O.V., Akhmedulova N.I., Gnidenko A.V. Design of clothes in the CAD system "Gracia". Tutorial. - Ivanovo: IGTA, 2004.- $124 \mathrm{~s}$

[4] Tashpulatova M. B., Bakhronova D. M. Main principles of computer-aided design of garments // Young Scientist. - 2016. №7. - p. 173-175.

[5] Mavlonova I.R. Software of the automated design system for garments // Young Scientist. - 2016. - №11. - p. 414-416.

[6] Mishenin O. A., Parkhomenko Yu.V. Improving the technological preparation of sewing production based on computer technology. “Textile industry”, 2008. - №3. - pp. 42-45.

[7] Eskin I.Yu. Auxiliary equipment for procurement and assembly sites of sewing production. Clothing industry, 2008. - №2. Pp. 2223.

[8] Petrov N.S. Equipment for sewing production at the XXVI Federal Wholesale Fair of Goods and Equipment for Textile and Light Industry. "Textile industry”, 2006. - №5. Pp. 26-29. 
[9] Cutting materials of garments. [Electronic resource]. - Access mode: http://www.znaytovar.ru/new3591.html, free. The title From the screen. - Yaz. russ

[10] Solomadina E.A., Zelinsky S.S. Functional methodology IDEF0 description of the subject area. Intellectual information technologies. Proceedings of the international scientific and practical youth conference. Penza, 2016. pp. 136-144.

[11] Novikova, TB, Nazarova, OB, Petelyak, V.E. IDEF0, DFD, IDEF3, Fishbone, FTA: Theory and Practice of Business Modeling. Tutorial. - Magnitogorsk: MSTU. G.I.Nosova, 2016. 97c.

[12] Taran V.N. Savchenko O.Yu. Maksimova-Fedortsova, IA Requirements analysis in the design of an information system. Sat mat. III All-Russian scientific-practical. Conferences with international participation dedicated to the 100th anniversary of the Crimean Fereralny University. V.I. Vernadsky. Information systems and technologies in modeling and management, Yalta. 2018. - p. 398-403.

[13] Frost, V.V. Designing new devices using the principles of universal design [Text] / V.V. Moroz, L.V. Baulin // Collection of theses of the international scientific-practical conference "Equal opportunities - a comfortable environment" (November 30 December 2, 2016): Sat. articles. Moscow 2016 - p. 50-51.

[14] Novikov. 10 robots that draw. [Electronic resource]. - Access mode: http://robotor.ru/2012/03/26/drawing-robots/, free. The title From the screen. - Yaz. russ

[15] Saaty T. Decision Making. Hierarchy analysis method. - M .: Radio and Communication, 1993. $-278 \mathrm{p}$.

[16] Structuralist. Introduction to the morphological methods of research and modeling knowledge of the subject area. [Electronic resource] - Access mode: http://www.structuralist.narod.ru/articles/morphmethod/morphmeth od.htm, free. Zagr from the screen. - Yaz. russ

[17] Chugunov D.S. Inverse-morphological approach to the synthesis of technical solutions: author. dis. Cand. those. sciences. Volgograd. state those. University, Volgograd, 2007.

[18] Medvedev A. Promising technologies and materials for developers of multilayer printed circuit boards // Technologies in the electronics industry. 2009. №1.

[19] A.E. Sabunin. Altium Designer. New solutions in the design of electronic devices / A.E. Sabunin. -M .: Solon-Press, 2009. -432 p.

[20] Shekhovtsov A.A., Karpova N.P. Additive technologies as a way to implement the concept of lean production // Scientific-methodical electronic journal concept. - 2015. - Vol. 13. - p. 141-145.

[21] Cutting of sewing materials. [Electronic resource]. - Access mode http://www.znaytovar.ru/new3591.html, free. Title from the screen. - Russ.

[22] Moroz, V.V. Designing new devices using the principles of universal design [Text] / V.V. Moroz, L.V. Baulina // Collected theses of the international scientific and practical conference: Sat. articles. Moscow, 2016. - P. 50-51.

[23] Novikov. 10 robots that draw. [Electronic resource]. - Access mode: http://robotor.ru/2012/03/26/drawing-robots/, free. Title from the screen. - Russ.

[24] Saati T. Decision-making. The method of analyzing hierarchies. Moscow: Radio and Communication, 1993. -278 p.

[25] Structuralist. Introduction to morphological methods of research and modeling knowledge of the subject area. [Electronic resource]. - Access mode: http://www.structuralist.narod.ru/articles/morphmethod/morphmeth od.htm, free. Title from the screen. - Russ. 